Usages et représentations du français hors de France

\title{
5 décembre 1987 - 14 décembre 2012
}

\section{Gérard Vigner}

\section{(2) OpenEdition}

\section{Journals}

\section{Édition électronique}

URL : https://journals.openedition.org/dhfles/3656

DOI : $10.4000 /$ dhfles.3656

ISSN : 2221-4038

\section{Éditeur}

Société Internationale pour l'Histoire du Français Langue Étrangère ou Seconde

\section{Édition imprimée}

Date de publication : 1 janvier 2013

Pagination : 11-13

ISSN : 0992-7654

\section{Référence électronique}

Gérard Vigner, « 5 décembre 1987 - 14 décembre 2012 ». Documents pour I'histoire du français langue étrangère ou seconde [En ligne], 50 | 2013, mis en ligne le 01 janvier 2017, consulté le 31 mars 2023. URL : http://journals.openedition.org/dhfles/3656 ; DOI : https://doi.org/10.4000/dhfles.3656 
5 décembre 1987

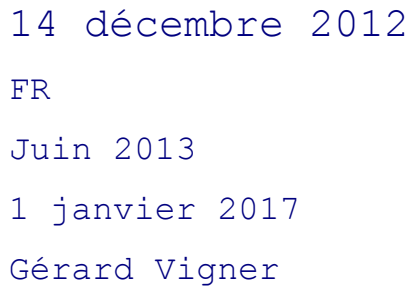

Le temps, ou plutôt le jeu des temporalités, semble s'imposer dans les consciences actuelles. Protestation contre un présent tout puissant qui absorbe tout, aussi bien les perspectives d'avenir que les linéaments d'un passé considéré comme révolu, donc sans incidence sur le présent. "Présentisme » que dénonce, ou au moins met en évidence, l'historien François Hartog; un présent vécu comme un motif d'enfermement qui condamne les acteurs du contemporain à errer dans un labyrinthe de ponctualités sans issue. Temps inaugural qui voit de la sorte surgir toute nouveauté sans la moindre antériorité annonciatrice. Comme si nous étions entrés dans la fin de l'histoire.

Que de grandes considérations me direz-vous pour l'approche de ces bien modestes outils de médiation entre les hommes que sont les méthodes, grandes ou petites, de langue. Méthodes nées de ce besoin, de cette envie de franchir les territoires de sa communauté et qui, à la surprise de bien d'entre nous, proviennent de fort lointains horizons. Ne découvre-t-on pas ainsi que, le $X V^{\mathrm{e}}$ siècle n'étant pas encore achevé, un certain William Caxton se préoccupait déjà de donner à son lecteur, anglais, les rudiments d'un échange, empreint de bien des civilités, dans la langue de son interlocuteur, français?

SALUTATIONS. HOW TO TAKE LEAVE OF FOLK. Je ne vous vey piecha. "I haue not seen you in longe tyme ». "Jay este longement hors du pays ». "I haue ben longe out of the contre ». " En quel pays?» «In what contre? « «Sire, ce seroit 'Syre, that shold be Trop a racompter ; Ouermoche for to telle ; Mais sil vous plaist aulcune chose But if you plaise ony thyng Que ie puisse fayre, That I may doo, Commandes le moy Commaunde it me Comme a celuy As to hym Qui volentiers le feroit'. That gladly shall doo it». «Sire, grand mercy 'Syre, gramercy De vous courtoyses parolles Of your courtoys wordes Et de vostre bonne volente; And of your good wyll; Dieu le vous mire !' God reward you !» « Dieu le me laisse deseruyr! 'God late me deserue it! Sachies certainement Knowe ye certaynely Que vous ne y estes That ye be not Point engaignies, Nothyng deceyued, Car ce vous feroye ie, For that wold I doo Pour vous et pour les vostres. For you and for youris. A Dieu vous comande. To god I you commaunde. Je prenge congie a vous' I take leue of you ». (William Caxton $1483: 51)$

De cette exploration des lointains, ne tirerions-nous que cette mise en évidence d'un fonds de permanence, sur la thématique bien rebattue du « tout est dit, et l'on vient trop tard depuis qu'il y a des hommes et qui pensent»? Un passé qui, à rebours,

\footnotetext{
${ }^{1}$ Dialogues in French and English, or 'A book for Travellers', or A Vocabulary', by William Caxton, adapted from a Fourteenth-Century Book of Dialogues in French and Flemish, edited from Caxton's printed text (about 1483), with introduction, notes, and word-lists, by Henry Bradley, M.A. London : published for the Early English Text Society, by Kegan Paul, Trench, Truebner \& Co, Itd. 1900. [http://www.hotfreebooks.com/book/Dialogues-in-French-and-English-William-Caxton.html].
} 
envelopperait le présent ? Et bien évidemment les historiens de la SIHFLES ne cessent de mettre à jour, qu'il s'agisse d'ouvrages de grammaires, de dictionnaires, de recueils de colloques, de modes d'organisation des cours, de trajectoires de carrières, avec celles de nos maîtres de langue devenus aujourd'hui plus familiers, toutes sortes d'outils, de démarches, d'objets de pensées qui témoignent de l'habileté, de l'ingéniosité de nos ancêtres. Nul progrès dans ces conditions, nulle avancée ? Serait-ce là tout le produit de notre travail ? À quoi peut bien servir l'histoire dans ces conditions?

Les choses sont bien plus subtiles qu'il n'y paraît, et l'on s'en serait bien douté s'agissant d'une matière aussi complexe. Dissiper l'opacité du passé, pour commencer, ce n'est pas rien. C'est même beaucoup, car le présent ainsi éclairé prend un autre profil et permet d'établir un lien différent entre l'ancien et le nouveau. Mais il en va bien plus qu'une simple exploration d'un passé qui s'opposerait de la sorte, très classiquement, au présent. Cette césure que pendant longtemps les historiens ont maintenue se fondait sur une sorte de commodité épistémologique. Seul un passé accompli était accessible à la connaissance. Une autre façon de se mettre à distance du présent, de le dévaluer ou de l'ignorer. Ne peut-on cependant reprendre ces différents points de vue, chacun légitime pour partie, et les inscrire dans une autre approche du travail historique, celle que nous suggérait Marc Bloch, pour lequel l'histoire se devait avant tout d'appréhender l'homme dans sa durée ? Passé et présent repris dans une saisie commune, l'événement et le temps long, les permanences et les évolutions, l'actuel dans la distance historique.

Et alors me direz-vous ? Est-il bien dans la vocation de l'histoire de parler du présent? Son projet n'est-il pas justement de s'éloigner de l'actuel, du hic et nunc ? Eh bien, non ! Faire de l'histoire c'est reconstruire les objets du passé avec les matériaux, les mots d'aujourd'hui, parce que de sourdes résonances existent entre ce passé lointain et les sensibilités qui sont les nôtres aujourd'hui.

Tel est bien notre projet, dans la modestie relative de son objet (que pèse en effet l'installation d'écoles congréganistes et leur façon d'enseigner le français dans les pays du Levant face à la tourmente née de la Première Guerre mondiale, à l'effondrement de l'empire ottoman et à toutes les répliques de guerre et de mort qu'il va faire naître ?), mais dans la ferme assurance d'un travail scientifiquement pertinent.

À travailler ainsi sur la longue durée, nous avons appris à mieux mesurer le rythme des évolutions, des changements, à mieux situer les points de tensions, d'innovation, à mieux appréhender le rôle joué par les acteurs et les institutions dans ce tissu de plus en plus dense d'échanges par les langues.

Telles pouvaient être les réflexions, entre autres, qui parcouraient cette assemblée du 14 décembre dernier lors de la célébration officielle de la création de la SIHFLES, un rappel de travaux et d'engagements inscrits dans une relation différente au temps de l'histoire. Un moment fort, dans la salle de la Grande bibliothèque du CIEP de Sèvres, institution qui avait justement vu naître la SIHFLES un jour de décembre 1987. Promesse avait été faite de s'engager sur un champ de recherche en ce temps-là encore peu exploré, promesse tenue au-delà de toutes les espérances. 